\title{
MOS surfaces: Medial Surface Transforms with Rational Domain Boundaries
}

\author{
Jiří Kosinka and Bert Jüttler \\ Johannes Kepler University, Institute of Applied Geometry, Altenberger Str. 69, \\ A-4040 Linz, Austria, \{Jiri.Kosinka|Bert. Juettler\}@jku.at
}

\begin{abstract}
We consider rational surface patches $\mathbf{s}(u, v)$ in the fourdimensional Minkowski space $\mathbb{R}^{3,1}$, which describe parts of the medial surface (or medial axis) transform of spatial domains. The corresponding segments of the domain boundary are then obtained as the envelopes of the associated two-parameter family of spheres. If the Plücker coordinates of the line at infinity of the (two-dimensional) tangent plane of $\mathbf{s}$ satisfy a sum-of-squares condition, then the two envelope surfaces are shown to be rational surfaces. We characterize these Plücker coordinates and analyze the case, where the medial surface transform is contained in a hyperplane of the four-dimensional Minkowski space.
\end{abstract}

\section{Introduction}

Planar and spatial domains can uniquely be represented by their medial axis transform or medial surface transform ${ }^{1}$. Various applications and computational aspects have been studied in Computer Vision, Computational Geometry and Computer Aided Design, see, e.g., $[1,4,6,7,11,16,19,21,22]$ and the references cited therein.

In the spatial case, the medial surface transform of a domain is a set of surface patches (and possibly curve segments, if canal surfaces are present) in 4dimensional $\left(x y z r^{-}\right)$space. Roughly speaking, each point on one of these curves and surfaces represents the center ( $x y z)$ and the radius $(r)$ of a sphere which is contained in the domain and touches the boundary in at least two points. This representation covers the structure of the domain and has many computational advantages. For instance, the operation of offsetting is equivalent to adding a constant to the radii, i.e., to a particular translation in the 4-dimensional space. Moreover, self-intersections of inner offsets can be detected by analyzing the radii of the spheres after the translation (see [4] for a discussion of the threedimensional case).

Rational representations of curves and surfaces (NURBS curves and surfaces) have become a universally accepted standard for Computer Aided Design.

\footnotetext{
${ }^{1}$ In the literature, the term medial axis transform is sometimes used both for twoand three-dimensional domains. In this paper we use medial surface transform for domains in three-dimensional space.
} 
However, this class is not closed under the operation of offsetting. As a remedy for this problem, the classes of Pythagorean hodograph curves and Pythagorean normal vector surfaces were introduced $[12,14,17,18]$. These curves and surfaces are characterized by the property that the length of the tangent or normal vector is a (possibly piecewise) rational function of the curve or surface parameters.

The notion of Pythagorean hodograph curves has been extended to the threedimensional Minkowski space $[2,4,5,16]$, simply by replacing the Euclidean length of the tangent vector (the hodograph) with the length defined by the indefinite inner product in Minkowski space. This led to the class of Minkowski Pythagorean hodograph curves. It is characterized by the property that the Minkowski length of the first derivative vector is a (piecewise) polynomial function of the curve parameter.

As observed in $[4,16]$, Minkowski Pythagorean hodograph curves in the three-dimensional Minkowski space are particularly well suited for representing the medial axis transforms of planar domains. If a segment of the medial axis transform is a Minkowski Pythagorean hodograph curve, then the associated pieces of the domain boundaries are segments of rational curves. This property is then shared by all offsets of the domain boundaries. Motivated by this observation, computational techniques for representing the medial axis transforms of general planar domains by Minkowski Pythagorean hodograph curves have been studied (see, e.g., [15]).

In the present paper, we generalize this idea to the class of two-dimensional surface patches in four-dimensional Minkowski space. These surface patches are used to describe segments of medial surface transforms. They are closely related to bisector surfaces between two given surfaces (which may degenerate into curves), cf. $[9,10]$.

The remainder of the paper is organized as follows. Section 2 summarizes some basic notions and facts concerning four-dimensional Minkowski geometry and medial surface transforms. The next section focuses on the local differential geometry of sheets od medial surface transforms and on the envelope formula for two-parameter families of spheres. This motivates the definition of MOS surfaces in Section 4. The fifth section analyzes the particular cases of MOS surfaces which are contained in hyperplanes. After briefly addressing the general case we conclude this paper.

\section{Preliminaries}

This section gives a summary of some basic results and concepts concerning Minkowski space $\mathbb{R}^{3,1}$ and medial surface transforms.

\subsection{Minkowski Space $\mathbb{R}^{3,1}$ and Homogeneous Coordinates}

The four-dimensional Minkowski space $\mathbb{R}^{3,1}$ is a four-dimensional real affine space equipped with the indefinite inner product

$$
\langle\mathbf{u}, \mathbf{v}\rangle=\mathbf{u}^{\top} G \mathbf{v}=u_{1} v_{1}+u_{2} v_{2}+u_{3} v_{3}-u_{4} v_{4}
$$


defined by the matrix

$$
G=\left(G_{i, j}\right)_{i, j=1,2,3,4}=\operatorname{diag}(1,1,1,-1),
$$

where $\mathbf{u}=\left(u_{1}, u_{2}, u_{3}, u_{4}\right)^{\top}, \mathbf{v}=\left(v_{1}, v_{2}, v_{3}, v_{4}\right)^{\top}$. The four axes spanned by the vectors $\mathbf{e}_{i}=\left(\delta_{i, 1}, \delta_{i, 2}, \delta_{i, 3}, \delta_{i, 4}\right)^{\top}, i=1, \ldots, 4$, will be called the $x^{-}, y-, z-$ and $r$-axis, respectively.

As the quadratic form associated with $G$ is not definite, the squared norm of a vector, which is defined by $\|\mathbf{v}\|^{2}=\langle\mathbf{v}, \mathbf{v}\rangle$, can be positive, negative or zero. Motivated by the special theory of relativity, one distinguishes three 'causal characters' of vectors: A vector $\mathbf{v}$ is said to be space-like if $\|\mathbf{v}\|^{2}>0$, time-like if $\|\mathbf{v}\|^{2}<0$, and light-like (or isotropic) if $\|\mathbf{v}\|^{2}=0$.

A hyperplane in Minkowski space is called space-, time- or light-like if the restriction of the quadratic form defined by $G$ on this plane is positive definite, indefinite nondegenerate or degenerate, respectively. The type of a hyperplane $\rho$ can be characterized by the Euclidean angle $\alpha$ included between $\rho$ and the $x y z$ hyperplane. For light-like hyperplanes, $\alpha=\frac{\pi}{4}$. Similarly one may distinguish between space-like, time-like and light-like 2-planes and lines.

A linear transform $L: \mathbb{R}^{3,1} \rightarrow \mathbb{R}^{3,1}$ is called a Lorentz transform if it maintains the Minkowski inner product, i.e. $\langle\mathbf{u}, \mathbf{v}\rangle=\langle L \mathbf{u}, L \mathbf{v}\rangle$ for all $\mathbf{u}, \mathbf{v} \in \mathbb{R}^{3,1}$. The set of all Lorentz transforms $\mathcal{L}=O(3,1)$, along with the composition, forms the Lorentz group. If the matrix $K=\left(k_{i, j}\right)_{i, j=1,2,3,4}$ represents a Lorentz transform, then its column vectors $\mathbf{k}_{1}, \mathbf{k}_{2}, \mathbf{k}_{3}$ and $\mathbf{k}_{4}$ satisfy $\left\langle\mathbf{k}_{i}, \mathbf{k}_{j}\right\rangle=G_{i, j}, i, j \in\{1,2,3,4\}$, i.e., they form an orthonormal basis of $\mathbb{R}^{3,1}$.

By introducing points at infinity we obtain the projective closure of the Minkowski space, which will be denoted by $\tilde{\mathbb{R}}^{3,1}$. Its points will be described using standard homogeneous coordinates $\tilde{\mathbf{p}}=\left(p_{0}: p_{1}: p_{2}: p_{3}: p_{4}\right)^{\top}$ satisfying

$$
1: x: y: z: r=p_{0}: p_{1}: p_{2}: p_{3}: p_{4} \text {. }
$$

Points at infinity (also called ideal points) are then characterized by $p_{0}=0$.

\subsection{Medial Surface Transform}

Consider a domain $\Omega \in \mathbb{R}^{3}$. Any sphere contained in $\Omega$ is said to be an inscribed sphere. The set of all inscribed spheres is partially ordered with respect to inclusion. An inscribed sphere is said to be maximal if it is not contained in any other inscribed sphere. The medial surface of $\Omega$ is the locus of all centers of maximal inscribed spheres.

The medial surface transform of the domain is obtained by adding the radius information to the medial surface. The medial surface transform consists of all points $(x, y, z, r)^{\top}$ in $\mathbb{R}^{3,1}$, where $(x, y, z)^{\top}$ is a point of the medial surface and $r$ is the radius of the maximal inscribed sphere centered at this point.

For a general domain with piecewise smooth boundary, the medial surface and the medial surface transform both consist of several components with different dimensions, see, e.g., [6]. Here we consider only two-dimensional components, which are called sheets. They correspond to the centers of inscribed spheres which 


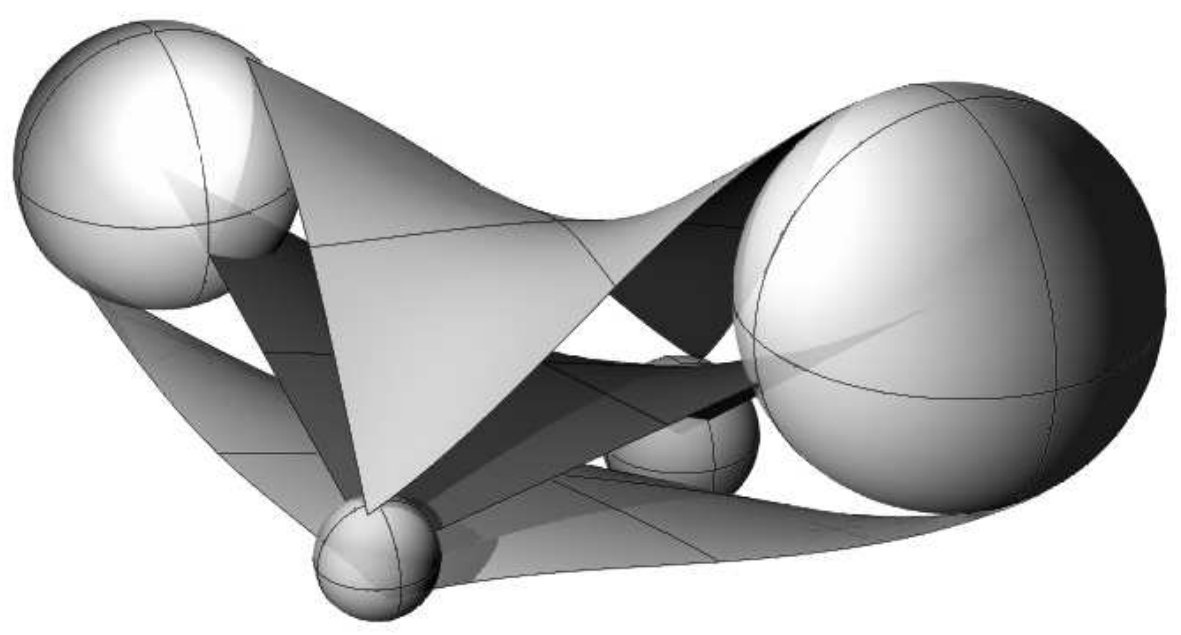

Fig. 1. A medial surface segment (dark grey) and the corresponding pieces of the boundary surface (light grey). In addition, four inscribed spheres are shown.

touch the domain boundary in two points. An example is shown in Fig. 1, which shows a quadrangular segment (patch) of the medial surface, along with the four inscribed spheres at the four vertices and the two corresponding quadrangular patches of the domain boundaries. If

$$
\mathbf{s}(u, v)=(x(u, v), y(u, v), z(u, v), r(u, v))^{\top},(u, v) \in D \subseteq \mathbb{R}^{2}
$$

is a regular surface patch in $\mathbb{R}^{3,1}$ which describes a sheet of the medial surface transform, then we can recover the associated part of the domain $\Omega$ as

$$
\Omega=\bigcup_{(u, v) \in D} B_{r(u, v)}(x(u, v), y(u, v), z(u, v))
$$

where $B_{r}(x, y, z)$ is the ball with center $(x, y, z)^{\top}$ and radius $r$. Its boundary $\partial \Omega$ contains the envelope of the spheres $\partial B_{r(u, v)}(x(u, v), y(u, v), z(u, v))$. In addition it contains segments of the canal surfaces which correspond to the boundary curves of $\mathbf{s}$, and segments of the spheres which correspond to the vertices of $\mathbf{s}$.

Remark 1. A two-parameter family of spheres is not always a valid medial surface transform. For instance, the families of spheres can be nested. 


\section{Tangent Planes of Sheets of Medial Surface Transforms and Envelope Formula}

We characterize the tangent 2-planes of sheets of the medial surface transform and derive the formula for computing the two envelope surfaces of the associated 2 -parameter sphere family.

\subsection{Tangent Planes of $2-$ surfaces in $\mathbb{R}^{3,1}$}

We consider a $C^{1}$ patch $\mathbf{s}:(u, v) \mapsto \mathbf{s}(u, v)$,

$$
\mathbf{s}(u, v)=(x(u, v), y(u, v), z(u, v), r(u, v))^{\top},
$$

of a 2 -surface in $\mathbb{R}^{3,1}$, where the parameters $(u, v)$ vary in a suitable parameter domain $D \subset \mathbb{R}^{2}$. The components of the associated first fundamental form are

$$
E=\left\langle\mathbf{s}_{u}, \mathbf{s}_{u}\right\rangle, \quad F=\left\langle\mathbf{s}_{u}, \mathbf{s}_{v}\right\rangle, \quad G=\left\langle\mathbf{s}_{v}, \mathbf{s}_{v}\right\rangle,
$$

with the partial derivative vectors ${ }^{2}$

$$
\mathbf{s}_{u}=\frac{\partial \mathbf{s}(u, v)}{\partial u} \quad \text { and } \quad \mathbf{s}_{v}=\frac{\partial \mathbf{s}(u, v)}{\partial v}
$$

At each regular point of $\mathbf{s}(u, v)$, the tangent plane is spanned by the two vectors $\mathbf{s}_{u}$ and $\mathbf{s}_{v}$. These vectors span a two-dimensional plane, which is embedded in the four-dimensional Minkowski space $\mathbb{R}^{3,1}$.

The intersection of a tangent $2-$ plane with the hyperplane $\omega$ at infinity defines a line $\nu=\nu(u, v)$. It is spanned by the two points

$$
\left(0: x_{u}: y_{u}: z_{u}: r_{u}\right)^{\top} \text { and }\left(0: x_{v}: y_{v}: z_{v}: r_{v}\right)^{\top} .
$$

We describe this line $\nu$ in the hyperplane at infinity using Plücker coordinates (see e.g. [20] for an introduction to line geometry):

Definition 1. For any regular surface patch $\mathbf{s}(u, v)$ in $\mathbb{R}^{3,1}$ we consider the homogeneous coordinates

$$
\left(p_{01}: p_{02}: p_{03}: p_{23}: p_{31}: p_{12}\right)^{\top}
$$

where

$$
\begin{gathered}
p_{01}=x_{u} y_{v}-y_{u} x_{v}, \quad p_{02}=x_{u} z_{v}-z_{u} x_{v}, \quad p_{03}=x_{u} r_{v}-r_{u} x_{v}, \\
p_{23}=z_{u} r_{v}-r_{u} z_{v}, \quad p_{31}=r_{u} y_{v}-y_{u} r_{v}, \quad p_{12}=y_{u} z_{v}-z_{u} y_{v} .
\end{gathered}
$$

They will be called the $\underline{\text { Plücker }}$ coordinates of the infinite line of the tangent plane, or PILT for short. The vector obtained by collecting them is called the PILT vector.

${ }^{2}$ Throughout the remainder of this paper, the subscripts $*_{u}, *_{v}$ denote partial derivatives of scalar or vector-valued quantities. 
Remark 2. 1. As for all Plücker coordinates, the six components of the PILT vector are not independent; they satisfy the Plücker condition

$$
p_{01} p_{23}+p_{02} p_{31}+p_{03} p_{12}=0 \text {. }
$$

2. The PILT vector can be seen as a generalization of the normal vector of a surface in three-dimensional Euclidean space. Indeed, the components of the PILT vector are the non-vanishing $3 \times 3$ subdeterminants of the matrix

$$
S=\left(\begin{array}{ccccc}
0 & x_{u} & y_{u} & z_{u} & r_{u} \\
0 & x_{v} & y_{v} & z_{v} & r_{v} \\
1 & 0 & 0 & 0 & 0
\end{array}\right)
$$

while the components of the normal vector of a surface $(X, Y, Z)$ can be obtained as the non-vanishing subdeterminants of the matrix

$$
R=\left(\begin{array}{cccc}
0 & X_{u} & Y_{u} & Z_{u} \\
0 & X_{v} & Y_{v} & Z_{v} \\
1 & 0 & 0 & 0
\end{array}\right),
$$

where the subscripts indicate (again) partial derivatives with respect to the surface parameters.

\subsection{Envelope Formula}

We consider the two-parameter family of spheres

$$
(X-x(u, v))^{2}+(Y-y(u, v))^{2}+(Z-z(u, v))^{2}-r^{2}(u, v)=0
$$

which correspond to the $C^{1}$ patch (6) of the medial surface transform. In addition to (15), a point $\mathbf{b}$ of one of the two envelopes satisfies the two equations

$$
\begin{gathered}
(X-x) \cdot x_{u}+(Y-y) \cdot y_{u}+(Z-z) \cdot z_{u}+r \cdot r_{u}=0 \\
(X-x) \cdot x_{v}+(Y-y) \cdot y_{v}+(Z-z) \cdot z_{v}+r \cdot r_{v}=0 .
\end{gathered}
$$

where $x, y, z$ and $r$ depend on $u, v$, and $\mathbf{b}=(X, Y, Z)$. Consequently, the coordinates of $\mathbf{b}$ can be found by intersecting the sphere (15) with two planes (16). This leads to a closed-form expression for the two branches $( \pm)$ of the envelope surface:

$$
\mathbf{b}^{ \pm}(u, v)=\hat{\mathbf{s}}(u, v)+\frac{r}{E_{0} G_{0}-F_{0}^{2}}\left(\mathbf{w} \pm \sqrt{E G-F^{2}} \cdot\left(\hat{\mathbf{s}}_{u} \times \hat{\mathbf{s}}_{v}\right)\right) .
$$

In this expression,

$$
\hat{\mathbf{s}}(u, v)=(x(u, v), y(u, v), z(u, v))^{\top}
$$

represents the corresponding medial surface, which is obtained by omitting the radius function in (6). 
The components $E, F, G$ of the first fundamental form of $\mathbf{s}(u, v)$, see (7), which are computed using the indefinite inner product (1) in $\mathbb{R}^{3,1}$. In addition, $E_{0}, F_{0}, G_{0}$ are the components of the first fundamental form of the corresponding medial surface (18), which are obtained by using the standard Euclidean inner product.

The coordinates of the vector $\mathbf{w}=\mathbf{w}(u, v)$ in (17) are polynomials of degree four in $x_{u}, y_{u}, z_{u}, r_{u}$ and $x_{v}, y_{v}, z_{v}, r_{v}$, where each coordinate consists of 8 terms. Also the other terms are quite complicated. For instance, the squared Minkowski area element $E G-F^{2}$ in (17) consists of 18 terms of degree 4 in the partial derivatives of $x, y, z$ and $r$.

A more compact representation can be derived by using the components of the PILT vector of the patch $\mathbf{s}(u, v)$ of the medial surface transform.

Lemma 1. The two envelope surfaces (17) of the two-parameter family of spheres corresponding to (6) can be rewritten as

$$
\mathbf{b}^{ \pm}(u, v)=\hat{\mathbf{s}}+\frac{r}{p_{01}^{2}+p_{02}^{2}+p_{12}^{2}}\left(\left(\begin{array}{c}
p_{02} p_{23}-p_{01} p_{31} \\
p_{23} p_{12}-p_{01} p_{03} \\
p_{31} p_{12}-p_{02} p_{03}
\end{array}\right) \pm \sqrt{C} \cdot\left(\begin{array}{c}
p_{12} \\
-p_{02} \\
p_{01}
\end{array}\right)\right),
$$

where

$$
C=E G-F^{2}=p_{01}^{2}+p_{02}^{2}-p_{03}^{2}-p_{23}^{2}-p_{31}^{2}+p_{12}^{2} .
$$

This observation can be verified by a direct computation.

Remark 3. 1. If the PILT vector vanishes, then the corresponding point of the medial surface transform is singular. Otherwise it is regular, i.e., $\mathbf{s}_{u}$ and $\mathbf{s}_{v}$ are linearly independent.

2. If $C=E G-F^{2}$ is positive, then the tangent 2 -plane is space-like and the surface $\mathbf{s}(u, v)$ is regular. Moreover, since $p_{01}^{2}+p_{02}^{2}+p_{12}^{2}>C$, the medial surface $\hat{\mathbf{s}}(u, v)$ is regular, too. Both envelope surfaces (19) are then welldefined.

3. If $C=E G-F^{2}=0$ and $\mathbf{s}$ is regular, then the 2 -surface $\mathbf{s}$ has a light-like tangent $2-$ plane. Due to the regularity of $\mathbf{s}$, not all components of the PILT vector vanish. Consequently, the medial surface is still regular, since

$$
E_{0} G_{0}-F_{0}^{2}=p_{01}^{2}+p_{02}^{2}+p_{12}^{2} .
$$

In this case, the envelope formula yields one point only.

4. If $C=E G-F^{2}$ is negative, then $\mathbf{b}^{ \pm}$is not defined. This characterizes the situation where the tangent 2 -plane is time-like.

5. Medial surface transforms with well defined envelope surface(s) will be called regular. From now on, we consider only regular medial surface transforms. These are medial surface transforms which have non-vanishing PILT vectors and either light-like or space-like tangent 2-planes only. This generalizes similar conditions for the medial axis transform of planar domains, which can be identified with a system of curves in Minkowski space $\mathbb{R}^{2,1}$. In that situation, curves with time-like tangents have to be excluded.

6. Lorentz transforms preserve the regularity of the PILT vector and the causal character of the tangent $2-$ plane. 


\section{Sheets of Medial Surface Transforms Defining Rational Envelopes}

In order to obtain rational envelopes, we introduce the notion of MOS surfaces and characterize their PILT vectors.

\subsection{MOS Surfaces}

Consider a medial surface transform (6), which is described by piecewise rational functions $x, y, z$ and $r$. We are particularly interested in the situation where the two envelope surfaces obtained from (19) are rational. This motivates the following definition:

Definition 2. Let $\mathbf{s}(u, v)=(x(u, v), y(u, v), z(u, v), r(u, v))^{\top}$ be a regular polynomial or rational medial surface transform in $\mathbb{R}^{3,1}$. We say that $\mathbf{s}(u, v)$ is a polynomial or rational medial surface transform which obeys the sumof-squares-condition (MOS surface for short), if there exists a bivariate polynomial or rational function $\sigma(u, v)$, such that the components of the PILT vector satisfy

$$
p_{01}^{2}+p_{02}^{2}-p_{03}^{2}-p_{23}^{2}-p_{31}^{2}+p_{12}^{2}=\sigma(u, v)^{2} .
$$

MOS surfaces generalize the notion of Minkowski Pythagorean hodograph curves, see $[4,16]$. If a segment of the medial axis transform of a planar domain is an Minkowski Pythagorean hodograph curve, then the corresponding segments of the domain boundary admit rational parameterization. Moreover, since the translations in Minkowski space preserve the hodographs (first derivative vectors), this is also the case for all offsets of the domain boundaries. In the surface case we get the following result.

Proposition 1. If a segment of the medial surface transform of a spatial domain is an MOS surface patch, then both the corresponding pieces of the boundary of the domain and their offsets admit a rational parametric representation.

Remark 4. Using the first fundamental form of $\mathbf{s}(u, v)$, the MOS condition in Definition 2 can be rewritten as

$$
E G-F^{2}=\left\langle\mathbf{s}_{u}, \mathbf{s}_{u}\right\rangle \cdot\left\langle\mathbf{s}_{v}, \mathbf{s}_{v}\right\rangle-\left\langle\mathbf{s}_{u}, \mathbf{s}_{v}\right\rangle^{2}=\sigma(u, v)^{2} .
$$

Consequently, the MOS surface is invariant with respect to Lorentz transforms. Moreover, the area (in the Minkowski sense) of a polynomial MOS is a (possibly piecewise) polynomial function of the surface parameters $u, v$.

\subsection{Characterizing the PILT Vectors of MOS Surfaces}

The 2-surface $\mathbf{s}(u, v)$ in $\mathbb{R}^{3,1}$ has the MOS property if its PILT vector simultaneously satisfies the MOS condition (22) and the Plücker condition (12). Consequently, in order to characterize MOS surfaces, we have to solve a system of two 


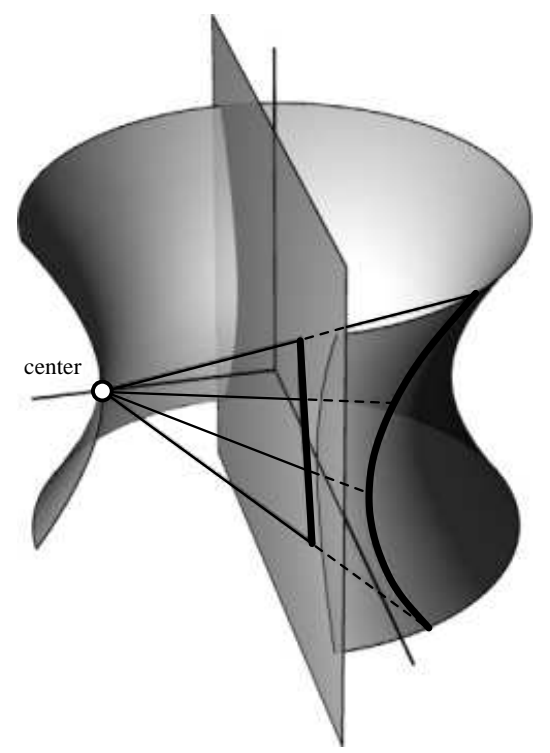

Fig. 2. A three-dimensional sketch of the central projection in $\mathbb{R}^{6}$. Rational curves in the (hyper)plane are projected onto the (hyper)surface.

quadratic equations. First, we use a central (or inverse stereographic) projection to solve (22). Let

$$
\left(p_{0}: p_{1}: p_{2}: p_{3}: p_{4}: p_{5}: p_{6}\right)^{\top}, p_{0} \neq 0
$$

be the homogeneous coordinates of a point in $\mathbb{R}^{6}$. The center of the projection is chosen at $(1:-1: 0: 0: 0: 0: 0)^{\top}$. We project points of the hyperplane $p_{1}=0$ onto the quadric hypersurface (22), see Fig. 2 for a sketch. This defines the mapping

$$
\left(\begin{array}{c}
p_{0} \\
0 \\
p_{2} \\
p_{3} \\
p_{4} \\
p_{5} \\
p_{6}
\end{array}\right) \mapsto\left(\begin{array}{c}
p_{0}^{2}+p_{2}^{2}-p_{3}^{2}-p_{4}^{2}-p_{5}^{2}+p_{6}^{2} \\
p_{0}^{2}-p_{2}^{2}+p_{3}^{2}+p_{4}^{2}+p_{5}^{2}-p_{6}^{2} \\
2 p_{0} p_{2} \\
2 p_{0} p_{3} \\
2 p_{0} p_{4} \\
2 p_{0} p_{5} \\
2 p_{0} p_{6}
\end{array}\right)
$$

Now we substitute the image (25) into Plücker's condition (12), which leads to

$$
p_{0}\left(-p_{4} p_{2}^{2}+p_{4} p_{3}^{2}+p_{4}^{3}+p_{4} p_{5}^{2}-p_{4} p_{6}^{2}+p_{0}^{2} p_{4}+2 p_{0} p_{2} p_{5}+2 p_{0} p_{3} p_{6}\right)=0 .
$$

This splits into the equation of a cubic hypersurface $\mathcal{C}$ and the hyperplane at infinity, both in $\mathbb{R}^{5}$. Since $p_{0}=0$ corresponds to the points at infinity, we omit 
the factor $p_{0}$ in $(26)$. As $\mathcal{C}$ has double points, we use again a central projection in order to obtain a parameterization.

Let $\left(r_{0}: r_{1}: r_{2}: r_{3}: r_{4}: r_{5}\right)^{\top}$ be the homogeneous coordinates of a point in $\mathbb{R}^{5}$. The surface $\mathcal{C}$ has a double point at $(0: 0: 0: 0: 1: 1)^{\top}$. We choose this point as the center of projection and map the points of the hyperplane determined by $r_{4}=0$ onto the cubic hypersurface given by the second factor in (26). A straightforward computation gives the cubic parameterization

$$
\left(\begin{array}{c}
r_{0} \\
r_{1} \\
r_{2} \\
r_{3} \\
0 \\
r_{5}
\end{array}\right) \mapsto\left(\begin{array}{c}
-2\left(r_{0} r_{1}+r_{0} r_{2}-r_{3} r_{5}\right) r_{0} \\
-2\left(r_{0} r_{1}+r_{0} r_{2}-r_{3} r_{5}\right) r_{1} \\
-2\left(r_{0} r_{1}+r_{0} r_{2}-r_{3} r_{5}\right) r_{2} \\
-2\left(r_{0} r_{1}+r_{0} r_{2}-r_{3} r_{5}\right) r_{3} \\
r_{3} r_{0}^{2}-r_{3} r_{1}^{2}+r_{3} r_{2}^{2}+r_{3}^{3}+2 r_{0} r_{2} r_{5}-r_{3} r_{5}^{2} \\
r_{3} r_{0}^{2}-r_{3} r_{1}^{2}+r_{3} r_{2}^{2}+r_{3}^{3}+r_{3} r_{5}^{2}-2 r_{5} r_{0} r_{1}
\end{array}\right)
$$

of $\mathcal{C}$. Finally we combine the parameterization (27) with the projection (25) and obtain (after omitting the common factor $\left.4 r_{0}\left(r_{0} r_{1}+r_{0} r_{2}-r_{3} r_{5}\right)\right)$ the final result:

Proposition 2. If $\mathbf{s}(u, v)$ is an MOS surface, then there exist five polynomials $r_{i}(u, v), i \in\{0,1,2,3,5\}$ and a polynomial $\lambda(u, v) \not \equiv 0$ such that the PILT vector of $\mathbf{s}(u, v)$ has the form

$$
\frac{1}{\lambda} \cdot\left(\begin{array}{c}
r_{1}\left(r_{0}^{2}-r_{1}^{2}+r_{3}^{2}-r_{5}^{2}-r_{1} r_{2}\right)+r_{2}\left(r_{0}^{2}+r_{2}^{2}+r_{3}^{2}+r_{5}^{2}+r_{1} r_{2}\right) \\
2\left(r_{0} r_{1}+r_{0} r_{2}-r_{3} r_{5}\right) r_{1} \\
2\left(r_{0} r_{1}+r_{0} r_{2}-r_{3} r_{5}\right) r_{2} \\
2\left(r_{0} r_{1}+r_{0} r_{2}-r_{3} r_{5}\right) r_{3} \\
-r_{3} r_{0}^{2}+r_{3} r_{1}^{2}-r_{3} r_{2}^{2}-r_{3}^{3}+r_{3} r_{5}^{2}-2 r_{0} r_{2} r_{5} \\
-r_{3} r_{0}^{2}+r_{3} r_{1}^{2}-r_{3} r_{2}^{2}-r_{3}^{3}-r_{3} r_{5}^{2}+2 r_{0} r_{1} r_{5}
\end{array}\right) .
$$

Indeed, the two central projections used for the parameterization are birational mappings, hence any given PILT vector satisfying (12) and (22) can be reproduced, but possibly with extraneous factors (which are taken care of by $\lambda$ ).

\section{MOS Surfaces in Hyperplanes}

In general, MOS surfaces spans the entire Minkowski space $\mathbb{R}^{3,1}$. As a special case, however, it can be contained in a hyperplane $\mathcal{H}$. This case will now be discussed in more detail.

\subsection{Classification}

The hyperplane $\mathcal{H}$ can be space-like, time-like or light-like. Using translations and Lorentz transforms, the hyperplane $\mathcal{H}$ can be mapped to one of the following three standard positions:

$\mathcal{H}$ is space-like $: r=0$,

$\mathcal{H}$ is time-like : $z=0$,

$\mathcal{H}$ is light-like $: z-r=0$. 
These three cases will be analyzed separately. We assume that the hyperplane $\mathcal{H}$ has the corresponding standard position.

Case 1: $\mathcal{H}$ is space-like. Since it lies in the hyperplane $r=0$, the equation of the MOS surface takes the form

$$
\mathbf{s}(u, v)=(x(u, v), y(u, v), z(u, v), 0)^{\top},
$$

i.e. it is contained in the Euclidean 3 -space $\mathbb{R}^{3}$, which is embedded into $\mathbb{R}^{3,1}$. The three components $p_{03}, p_{31}, p_{23}$ of the PILT vector vanish. The remaining components are equal to the components of the cross product $\hat{\mathbf{s}}_{u} \times \hat{\mathbf{s}}_{v}$ of the medial surface (18). The MOS condition (22) simplifies to

$$
p_{01}^{2}+p_{02}^{2}+p_{12}^{2}=\sigma(u, v)^{2} .
$$

Consequently, these surfaces are PN (Pythagorean normal) surfaces as introduced in [18]. These surfaces are equipped with a rational field of unit normals. Therefore, the offset surfaces are again rational.

In the standard position, both envelope surfaces (17) degenerate into the medial surface. If the MOS surface lies in a plane of the form $r=c$, where $c$ is a constant, then the two envelopes are the offset surfaces at distance $c$. MOS surfaces in general space-like hyperplanes can be obtained by first embedding PN surfaces in the hyperplane $\mathcal{H}$ of $\mathbb{R}^{3,1}$ and then applying Lorentz transforms.

Case 2: $\mathcal{H}$ is time-like. Since it lies in the hyperplane $z=0$, the equation of the MOS surface takes the form

$$
\mathbf{s}(u, v)=(x(u, v), y(u, v), 0, r(u, v))^{\top},
$$

i.e. it is contained in the Minkowski 3-space $\mathbb{R}^{2,1}$, which is embedded into $\mathbb{R}^{3,1}$. The three components $p_{02}, p_{12}, p_{23}$ of the PILT vector vanish. The remaining three components are equal to the coordinates of the Minkowski cross product ${ }^{3}$ $\tilde{\mathbf{s}}_{u} \bowtie \tilde{\mathbf{s}}_{v}$, where

$$
\tilde{\mathbf{s}}=(x(u, v), y(u, v), r(u, v))^{\top} .
$$

The MOS condition (22) simplifies to

$$
p_{03}^{2}+p_{31}^{2}-p_{01}^{2}=\left\|\tilde{\mathbf{s}}_{u} \bowtie \tilde{\mathbf{s}}_{v}\right\|^{2}=-\sigma(u, v)^{2} .
$$

Consequently, the Minkowski norm of the Minkowski cross product $\tilde{\mathbf{s}}_{u} \bowtie \tilde{\mathbf{s}}_{v}$ is minus the square of a polynomial. The vector $\tilde{\mathbf{s}}_{u} \bowtie \tilde{\mathbf{s}}_{v}$ is therefore time-like.

The medial surface of the MOS surface (32) is always contained in the plane $z=0$. Consequently, the two envelopes of the associated sphere family are symmetric with respect the plane $z=0$.

MOS surfaces in general time-like hyperplanes can be obtained by applying Lorentz transforms to an MOS surface of the form (32). These Lorentz transforms do not preserve the planarity of the medial surface.

\footnotetext{
$\overline{{ }^{3}\left(u_{1}, u_{2}, u_{3}\right)^{\top}} \bowtie\left(v_{1}, v_{2}, v_{3}\right)^{\top}=\left(u_{2} v_{3}-u_{3} v_{2}, u_{3} v_{1}-u_{1} v_{3},-u_{1} v_{2}+u_{2} v_{1}\right)^{\top}$, cf. [15]
} 
Case 3: $\mathcal{H}$ is light-like. In the standard position, the equation of the MOS surface takes the form

$$
\mathbf{s}(u, v)=(x(u, v), y(u, v), z(u, v), z(u, v))^{\top} .
$$

Consequently, in this case, $p_{23}=0, p_{02}=p_{03}$ and $p_{12}=-p_{31}$. The MOS condition simplifies to $p_{01}^{2}=\sigma(u, v)^{2}$. Thus, any rational 2-surface lying in a light-like hyperplane is an MOS surface. One of the two envelopes of the associated family of spheres is then contained in the plane which is obtained by intersecting the light-like hyperplane with the hyperplane $r=0$, where the latter one is identified with the Euclidean space.

\subsection{Construction}

MOS surfaces in space-like or time-like hyperplanes $\mathcal{H}$ can be generated with the help of the following construction.

1. Choose a suitable field $\mathbf{N}(u, v)$ of normal vectors. More precisely, find three bivariate polynomials $p_{01}, p_{02}, p_{12}$ or $p_{03}, p_{31}, p_{01}$ in $u, v$, such that the conditions (31) or (34) are satisfied, respectively. These polynomials can be found by parameterizing the corresponding quadrics. This can be done, e.g., by stereographic projection, or using the method of generalized stereographic projection [8], or its equivalent formulation via Pythagorean hodograph representation maps [5].

2. Find a polynomial or rational surface $\mathbf{s}$ in the standard position of the given hyperplane $\mathcal{H}$, such that the first partial derivatives satisfy

$$
\left\langle\mathbf{N}(u, v), \mathbf{s}_{u}(u, v)\right\rangle \equiv 0, \quad\left\langle\mathbf{N}(u, v), \mathbf{s}_{v}(u, v)\right\rangle \equiv 0
$$

where the inner product $\langle.,$.$\rangle is obtained by restricting the inner product of$ $\mathbb{R}^{3,1}$ to the standard position of $\mathcal{H}$. If these two conditions are satisfied, then the two vector fields $\mathbf{N}(u, v)$ and $\mathbf{s}_{u}(u, v) \times \mathbf{s}_{v}(u, v)$ are linearly dependent for all $u, v$, and the latter one is therefore obtained by multiplying $\mathbf{N}(u, v)$ by a rational function.

- A polynomial surface satisfying (36) can be obtained by comparing the coefficients. If $\mathbf{N}$ has degree $k$ and $\mathbf{s}$ has degree $n$ in $u, v$, then the two equations lead to $2\left(\begin{array}{c}n+k+1 \\ 2\end{array}\right)$ equations for the $3\left(\begin{array}{c}n+2 \\ 2\end{array}\right)-3$ coefficients of $\mathbf{s}$. If $n$ is sufficiently large, then the number of unknowns exceeds the number of linear equations in the homogeneous linear system and nontrivial solutions are therefore guaranteed to exist (for $k=2$ is $n=5$ sufficiently large).

- A rational surface satisfying (36) can be generated by computing the envelope of the two-parameter family of planes

$$
G(u, v ; \mathbf{x})=\langle\mathbf{N}(u, v), \mathbf{x}\rangle-h(u, v)=0,
$$

where $\mathrm{h}(\mathrm{u}, \mathrm{v})$ is another rational function. It can be found by solving the three linear equations $G=G_{u}=G_{v}=0$ for $\mathbf{x}$. This approach has been used in [18] for the construction of PN surfaces. 
3. Finally, use a Lorentz transform, in order to map the standard position of the hyperplane into the given general position $\mathcal{H}$.

\subsection{Example}

We present an MOS surface which is contained in a time-like hyperplane.

Example 1. In step 1, we choose the normal vectors

$$
\mathbf{N}(u, v)=\left(2 u v,-2 u, u^{2}+1+v^{2}\right)^{\top},
$$

which satisfy

$$
-\mathbf{N}_{1}^{2}-\mathbf{N}_{2}^{2}+\mathbf{N}_{3}^{2}=\left(u^{2}-v^{2}-1\right)^{2} .
$$

In step 2 , in order to generate a polynomial surface, we choose $n=3$. By comparing the coefficients we obtain a system of $2\left(\begin{array}{c}6 \\ 2\end{array}\right)=30$ linear equations for the $3\left(\begin{array}{l}5 \\ 2\end{array}\right)=30$ unknown coefficients. A particular solution is the polynomial MOS surface

$$
\mathbf{s}(u, v)=\left(\begin{array}{c}
-15 u v^{2}+u^{3}-6 u v+\frac{3}{2} v^{2}-3 u+2 v \\
3 u^{3} v-9 u v^{3}-3 u v^{2}+v^{3}+u^{3}+v^{2}+3 u v+3 u \\
0 \\
-6 u^{2} v-3 u^{2}+1
\end{array}\right) .
$$

Fig. 3a shows the medial surface and the associated rational domain boundaries. Finally, in step 3, we apply the Lorentz transform

$$
L=\left(\begin{array}{cccc}
1 & 0 & 0 & 0 \\
0 & 1 & 0 & 0 \\
0 & 0 & \cosh \beta & \sinh \beta \\
0 & 0 & \sinh \beta & \cosh \beta
\end{array}\right)
$$

with $\beta=\ln 5$. This leads to the polynomial MOS surface

$$
L \mathbf{s}(u, v)=\left(\begin{array}{c}
-15 v^{2} u+u^{3}-6 v u+\frac{3}{2} v^{2}-3 u+2 v \\
3 v u^{3}-9 v^{3} u-3 v^{2} u+v^{3}+u^{3}+v^{2}+3 v u+3 u \\
\frac{1}{5}\left(-72 v u^{2}-36 u^{2}+12\right) \\
\frac{1}{5}\left(-78 v u^{2}-39 u^{2}+13\right)
\end{array}\right)
$$

in the time-like hyperplane $13 z=12 r$, see Fig. 3b. It corresponds to two rational domain boundaries with a non-planar medial (bisector) surface.

\section{General MOS Surfaces}

While MOS surfaces lying in hyperplanes are now fully understood, the situation is less clear in the general case. 


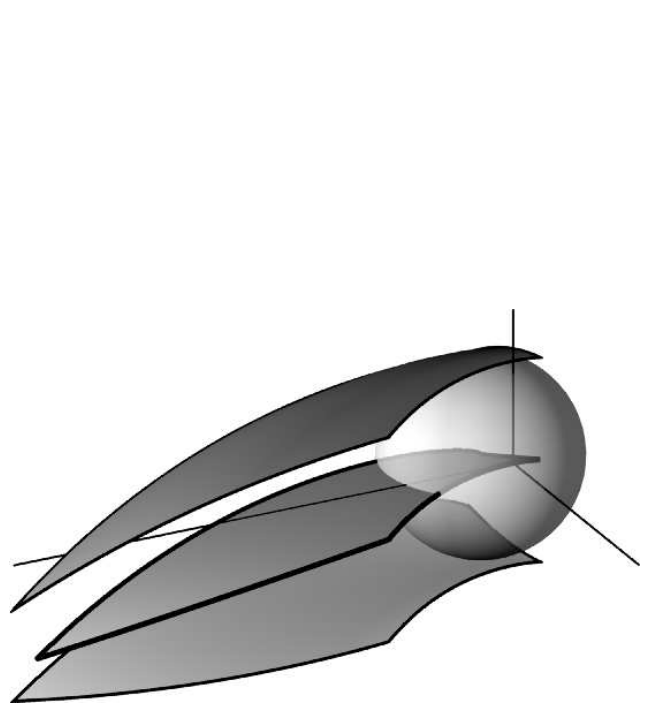

(a)

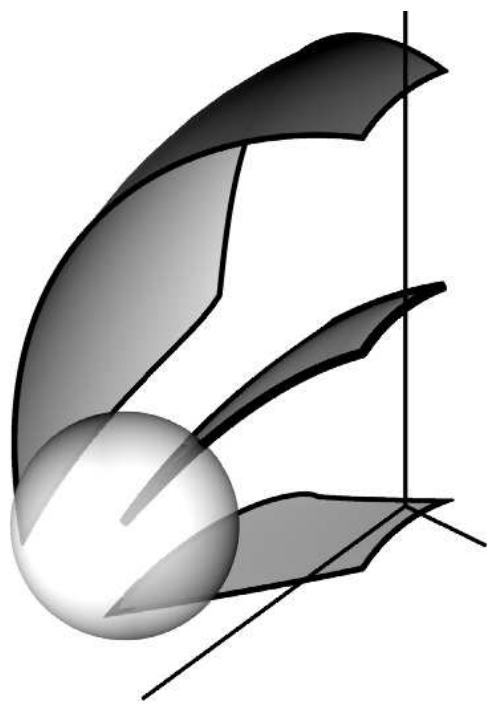

(b)

Fig. 3. Example 1: The MOS surface $\mathbf{s}(u, v)$ in the time-like plane $z=0$ (a) and the same MOS surface after applying the Lorentz transform (41). In both cases, one of the 'corner' spheres is also shown.

\subsection{Existence and Examples}

Still we have the following result.

Proposition 3. There exist both polynomial and rational MOS surfaces.

Proof. Consider a non-planar polynomial Minkowski Pythagorean hodograph curve in $\mathbb{R}^{2,1}$ and the associated planar domain $\Omega_{0}$ in the $x y$-plane. Assume that the $y$-axis does not intersect $\Omega_{0}$. Then the spatial domain obtained by rotating $\Omega_{0}$ around the $y$-axis has an medial surface transform which can be described by a rational MOS surface, and the spatial domain obtained by translating $\Omega_{0}$ parallel to the $z$-axis has an medial surface transform which can be described by a polynomial MOS surface.

Example 2. Consider a torus and a point $\mathbf{S}$ in $\mathbb{R}^{3}$. Their bisector surface, along with the associated radius function, defines a rational MOS surface. For the sake of simplicity, we choose the radii of the torus to be 1 and 2 , respectively. Moreover, we assume that the center of the torus is at $\mathbf{S}=(0,0,0)^{\top}$ (see Fig. 4). A rational parameterization of the torus is

$$
\mathbf{T}(u, v)=\left(\frac{-\left(3+v^{2}\right)\left(-1+u^{2}\right)}{\left(1+v^{2}\right)\left(1+u^{2}\right)}, \frac{2\left(3+v^{2}\right) u}{\left(1+v^{2}\right)\left(1+u^{2}\right)}, \frac{2 v}{\left(1+v^{2}\right)}\right)^{\top} .
$$




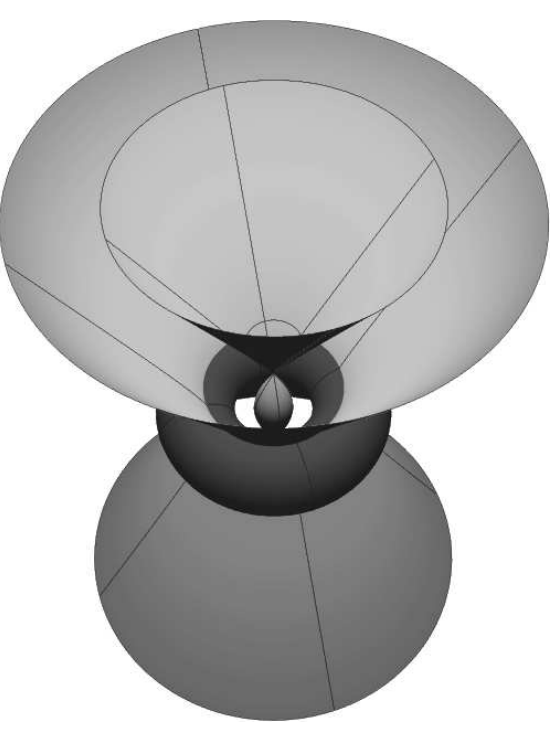

a)

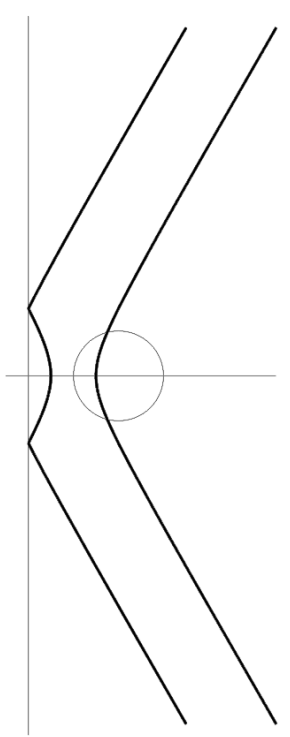

b)

Fig. 4. Example 2: Bisector surface of a torus (dark) and a point (center of the torus). a) $3 \mathrm{D}$ view, b) The half-meridians of the bisector surface and of the torus.

Using the method described in [10] we compute the rational parameterization of the bisector $\hat{\mathbf{s}}(u, v)$ by solving the following system of linear equations for the coordinates of the bisector surface,

$$
\begin{aligned}
& \left\langle\hat{\mathbf{s}}(u, v)-\mathbf{T}(u, v), \mathbf{T}_{u}\right\rangle_{E}=0, \\
& \left\langle\hat{\mathbf{s}}(u, v)-\mathbf{T}(u, v), \mathbf{T}_{v}\right\rangle_{E}=0, \\
& 2\langle\hat{\mathbf{s}}(u, v), \mathbf{T}(u, v)-\mathbf{S}\rangle_{E}=\langle\mathbf{T}(u, v), \mathbf{T}(u, v)\rangle_{E}-\langle\mathbf{S}, \mathbf{S}\rangle_{E} .
\end{aligned}
$$

Here, $\langle., .\rangle_{E}$ denotes the Euclidean inner product. The bisector surface is

$$
\hat{\mathbf{s}}(u, v)=\left(\frac{\left(1-u^{2}\right)\left(-9+v^{2}\right)}{2\left(1+u^{2}\right)\left(v^{2}-3\right)}, \frac{u\left(-9+v^{2}\right)}{\left(1+u^{2}\right)\left(v^{2}-3\right)}, \frac{3 v}{\left(v^{2}-3\right)}\right)^{\top} .
$$

It is a surface of revolution around the $z$-axis. This surface and its meridian curve is shown in Figure 4. The corresponding rational radius function can be computed by evaluating the Euclidean distance

$$
\|\hat{\mathbf{s}}(u, v)-\mathbf{S}\|_{E}=\frac{9+v^{2}}{2\left(v^{2}-3\right)} .
$$

This leads to the medial surface transform

$$
\mathbf{s}(u, v)=\left(\frac{\left(1-u^{2}\right)\left(-9+v^{2}\right)}{2\left(1+u^{2}\right)\left(v^{2}-3\right)}, \frac{u\left(-9+v^{2}\right)}{\left(1+u^{2}\right)\left(v^{2}-3\right)}, \frac{3 v}{v^{2}-3}, \frac{9+v^{2}}{2\left(v^{2}-3\right)}\right)^{\top} .
$$


In order to verify that this is indeed an MOS surface, we evaluate

$$
\left\langle\mathbf{s}_{u}, \mathbf{s}_{u}\right\rangle \cdot\left\langle\mathbf{s}_{v}, \mathbf{s}_{v}\right\rangle-\left\langle\mathbf{s}_{u}, \mathbf{s}_{v}\right\rangle^{2}=\frac{9(v-3)^{2}(v+3)^{2}}{\left(1+u^{2}\right)^{2}\left(v^{2}-3\right)^{4}}
$$

and obtain the square of a rational function. Finally, we show that $\mathbf{s}(u, v)$ is not contained in a hyperplane. Indeed, the five points of $\mathbf{s}(u, v)$ with the parameter values

$$
(u, v) \in\{(-1,0),(-1,1),(0,1),(1,-1),(1,1)\}
$$

can be shown to be in general position.

For the sake of simplicity we chose a special mutual position of the torus and the point. However, it can be shown that for any radii of the torus and any position of the point $\mathbf{S}$, the bisector surface along with the associated radius function defines an MOS surface. Since offsetting corresponds to a translation in Minkowski space, this is also true for sphere/torus bisectors. By applying general Lorentz transforms, this even applies to bisectors of certain cyclides and spheres.

\subsection{Towards the Construction of General MOS Surfaces}

If the tangent 2-planes of two surfaces $\mathbf{s}=(x, y, z, r)$ and $\mathbf{s}^{\prime}=\left(x^{\prime}, y^{\prime}, z^{\prime}, r^{\prime}\right)$, which describe medial surface transforms of spatial domains, share the same line at infinity, then the two $4 \times 3$ matrices

$$
\left(\mathbf{s}_{u}, \mathbf{s}_{u}^{\prime}, \mathbf{s}_{v}^{\prime}\right) \text { and }\left(\mathbf{s}_{v}, \mathbf{s}_{u}^{\prime}, \mathbf{s}_{v}^{\prime}\right)
$$

have both rank 2 . By evaluating the $3 \times 3$ subdeterminants, this can be characterized by two times two equations, which are linear in the partial derivatives of $\mathbf{s}$. These equations involve the components of the PILT vector of $\mathbf{s}^{\prime}$, since this vector consists of the $2 \times 2$ subdeterminants of the last two columns.

This may potentially be useful for constructing MOS surfaces with a given field of PILT vectors. Recall that these vectors of general MOS surfaces can be obtained from Proposition 2. Once a PILT vector has been found, one may try to find a matching MOS surface. In order to find a polynomial MOS surface which is compatible with a given PILT vector one may generate a system of linear equations by comparing the coefficients.

A similar approach may be used for rational MOS surfaces, leading to a system of non-linear equations.

However, even in the polynomial case, the number of equations exceeds the number of free parameters, and solutions do not exist in general. This is due to the fact that the number of equations grows roughly with the same speed (it is described by a quadratic polynomial in $n$ with the leading term is $4 n^{2}$, where $n$ is the degree of the unknown MOS surface) as the number of free parameters (it is also a polynomial in $n$ with the same leading term $4 n^{2}$ ).

This is a major difference to the hyperplanar case. In that case, the number of equations is described by a polynomial with the leading term $2 n^{2}$, while the number of free parameters is a polynomial with the leading term $3 n^{2}$. Consequently, if $n$ is large enough, then more free parameters than equations are present. Unfortunately, this argument does not apply to the general case. 


\section{Conclusion}

Minkowski Pythagorean hodograph curves can be used to represent the medial axis transform of planar domains $[4,15,16]$. In this paper we generalized this notion to the case of surfaces. We introduced the new class of MOS surfaces, which are special 2-surfaces in the four-dimensional Minkowski space $\mathbb{R}^{3,1}$. By using them to describe segments of the medial surface transform one obtains rational segments of the associated domain boundary.

MOS surfaces can be characterized by the so-called PILT vector, which represents the direction of the tangent $2-$ plane. Using this vector provides a particularly compact representation of the envelope formula.

The case of MOS surfaces in hyperplanes was studied in some detail. MOS surfaces in space-like planes are equivalent to PN surfaces, see $[17,18]$. MOS surfaces in time-like planes seem to possess no counterpart in the existing literature.

The detailed investigation of MOS surfaces poses many challenging problems, such as the construction of general MOS surfaces. This may lead to a systematic analysis of curve and surface pairs with rational bisector surfaces (cf. $[9,10])$, where the associated radius functions are rational too.

Acknowledgment The authors were supported by the Austrian Science Fund (FWF) through project P17387-N12. Special thanks go to Josef Schicho for his help with the parameterization of the PILT vectors of MOS surfaces. The authors would like to thank the referees for their comments which have helped to improve the first version of this paper.

\section{References}

1. Amenta, N., Choi, S., Kolluri, R. K.: The power crust, unions of balls, and the medial axis transform, Comput. Geom. 19 (2001), 127-153.

2. Cho H. Ch., Choi, H. I., Kwon S.-H., Lee D. S., Wee N.-S.: Clifford algebra, Lorentzian geometry and rational parametrization of canal surfaces, Computer Aided Geometric Design 21 (2004) 327-339

3. Choi, H. I., Choi, S. W., Moon, H. P.: Mathematical theory of medial axis transform, Pacific J. Math. 181 (1997) 57-88

4. Choi, H. I., Han, Ch. Y., Moon, H. P., Roh, K. H., Wee, N. S.: Medial axis transform and offset curves by Minkowski Pythagorean hodograph curves, Computer-Aided Design 31 (1999) 59-72.

5. Choi, H. I., Lee, D. S., Moon, H. P.: Clifford algebra, spin representation and rational parameterization of curves and surfaces, Advances in Computational Mathematics 17 (2002) 5-48

6. Culver, T., Keyser, J., Manocha, D.: Exact computation of the medial axis of a polyhedron, Comput. Aided Geom. Des. 21 (2004), 65-98.

7. Dey, T. K., Zhao, W.: Approximating the medial axis from the Voronoi diagram with a convergence guarantee, in Algorithms - ESA 2002 (Möhring, R., et al., eds.), Springer, LNCS 2461, 387-398, Berlin 2002. 
8. Dietz, R., Hoschek, J., Jüttler, B.: An algebraic approach to curves and surfaces on the sphere and on other quadrics. Comput. Aided Geom. Des. 10 (1993), 211-229.

9. Elber, G., Kim, M.-S.: The Bisector Surface of Rational Space Curves, ACM Transactions on Graphics 17 (1998) 32-49

10. Elber, G., Kim, M.-S.: Computing Rational Bisectors of Point/Surface and Sphere/Surface Pairs, IEEE Comput. Graph. Appl. 19 (1999) 76-81

11. Giblin, P., Kimia, B. B.: Transitions of the 3D medial axis under a one-parameter family of deformations, in Proc. ECCV 2002 (Heyden, A., et al., eds.), Springer, LNCS 2351, 718-734, Berlin 2002.

12. Farouki, R. T.: Pythagorean-hodograph curves, Handbook of Computer Aided Geometric Design (J. Hoschek, G. Farin \& M.-S. Kim, eds.) (2002), Elsevier 405427

13. Farouki, R. T., al-Kandari, M., Sakkalis, T: Hermite interpolation by rotationinvariant spatial Pythagorean-hodograph curves, Advances in Computational Mathematics 17 (2002) 369-383

14. Farouki, R. T., Sakkalis T.: Pythagorean hodographs, IBM Journal of Research and Development 34 (1990) 736-752

15. Kosinka, J., Jüttler, B.: $G^{1}$ Hermite Interpolation by Minkowski Pythagorean hodograph cubics, Computer Aided Geometric Design 23 (2006), 401-418.

16. Moon, H. P.: Minkowski Pythagorean hodographs, Computer Aided Geometric Design 16 (1999) 739-753

17. Peternell, M., Pottmann, H.: A Laguerre Geometric Approach to Rational Offsets, Computer Aided Geometric Design 15 (1998), 223-249.

18. Pottmann, H.: Rational curves and surfaces with rational offsets, Computer Aided Geometric Design 12 (1995) 175-192

19. Pottmann, H., Peternell, M.: Applications of Laguerre geometry in CAGD, Computer Aided Geometric Design 15 (1998), 165-186.

20. Pottmann, H., Wallner, J.: Computational Line Geometry, Springer, Berlin 2001.

21. Ramanathan, M., Gurumoorthy, B.: Constructing medial axis transform of extruded and revolved 3D objects with free-form boundaries, Computer-Aided Design 37 (2005), 1370-1387.

22. Sampl, P.: Medial axis construction in three dimensions and its application to mesh generation. Eng. Comput. 17, 234-248 (2001).

23. Walrave, J.: Curves and surfaces in Minkowski space, 1995, Doctoral thesis, K. U. Leuven, Fac. of Science, Leuven. 